\title{
Neural Substrates for Behaviorally Conditioned Immunosuppression in the Rat
}

\author{
Gustavo Pacheco-López, ${ }^{1,3}$ Maj-Britt Niemi, ${ }^{3}$ Wei Kou, ${ }^{1}$ Margarete Härting, ${ }^{1}$ Joachim Fandrey, ${ }^{2}$ and \\ Manfred Schedlowski ${ }^{3}$ \\ Departments of ${ }^{1}$ Medical Psychology and ${ }^{2}$ Physiology, University Duisburg-Essen, D-45122 Essen, Germany, and ${ }^{3}$ Division of Psychology and Behavioral \\ Immunobiology, Swiss Federal Institute of Technology, ETH-Zurich CH-8603, Switzerland
}

\begin{abstract}
We have previously demonstrated behaviorally conditioned immunosuppression using cyclosporin A as an unconditioned stimulus and saccharin as a conditioned stimulus. In the current study, we examined the central processing of this phenomenon generating excitotoxic lesions before and after acquisition to discriminate between learning and memory processes. Three different brain areas were analyzed: insular cortex (IC), amygdala (Am), and ventromedial nucleus of the hypothalamus (VMH). The results demonstrate that IC lesions performed before and after acquisition disrupted the behavioral component of the conditioned response (taste aversion). In contrast, Am and VMH lesions did not affect conditioned taste aversion. The behaviorally conditioned suppression of splenocyte proliferation and cytokine production (interleukin-2 and interferon- $\gamma$ ) was differentially affected by the excitotoxic lesions, showing that the IC is essential to acquire and evoke this conditioned response of the immune system. In contrast, the Am seems to mediate the input of visceral information necessary at the acquisition time, whereas the VMH appears to participate within the output pathway to the immune system necessary to evoke the behavioral conditioned immune response. The present data reveal relevant neural mechanisms underlying the learning and memory processes of behaviorally conditioned immunosuppression.
\end{abstract}

Key words: behavioral conditioning; lymphocyte proliferation; cytokines; insular cortex; ventromedial nucleus of the hypothalamus; amygdala; cyclosporin A

\section{Introduction}

Experimental evidence demonstrates bidirectional communication among the nervous, the endocrine, and the immune systems (Elmquist et al., 1997; Dantzer et al., 2000; Tracey, 2002). One of the most exquisite examples of brain-immune interactions is the behavioral conditioning of immune functions. The conditioning of both cellular and humoral immune responses has been well documented (Ader and Cohen, 2000; Exton et al., 2001). However, to date, little advance has been made to understand the central processing underlying the conditioning of immune functions.

We have developed a conditioning model to reenlist specific immune changes produced by administration of the immunosuppressive drug cyclosporin A (CsA) (Exton et al., 2000b, 2001). Our approach involves the pairing of a novel taste [saccharin $(\mathrm{Sac})]$ as the conditioned stimulus (CS) with CsA as the unconditioned stimulus (UCS). When the CS is represented, animals develop a conditioned taste aversion (CTA) (Garcia et al., 1985). Concomitantly, these animals present a suppression of the lym-

\footnotetext{
Received June 16, 2004; revised Dec. 28, 2004; accepted Jan. 14, 2005.

This work was supported by German Academic Exchange Service fellowships to G.P.-L. and by German Research Foundation Grants Deutsche Forschungsgemeinschaft (DFG) SCHE-341/9-1 and DFG SCHE-341/9-2.

Correspondence should be addressed to Dr. Gustavo Pacheco-López, Division of Psychology and Behavioral Immunology, Swiss Federal Institute of Technology, ETH-Zurich CH-8603, Switzerland. E-mail: gustavo.pacheco@ifv.gess.ethz.ch.

D0I:10.1523/JNEUROSCI.4230-04.2005

Copyright $\odot 2005$ Society for Neuroscience $\quad$ 0270-6474/05/252330-08\$15.00/0
}

phocyte responsiveness mimicking the immunosuppressive effects of CsA, also reflected at interleukin-2 (IL-2) and interferon- $\gamma($ IFN- $\gamma$ ) mRNA expression and synthesis (Exton et al., 1998b,c, 2000a). Of note, conditioned effects on the immune system are clinically relevant, because a significant prolongation of allograft survival (Exton et al., 1998a) and reduced delayedtype hypersensitivity response have been reported (Exton et al., 2000a). Importantly, we previously revealed that behaviorally conditioned immunosuppression is not related to hypothalamicpituitary-adrenal axis activation and is merely mediated via neural innervation of the spleen, via noradrenalin- and $\beta$-adrenoceptordependent mechanisms (Exton et al., 1999, 2002).

Thus far, however, only a limited number of studies have attempted to analyze the central mechanisms responsible for the conditioning of immune functions (Ramírez-Amaya et al., 1996, 1998; Hsueh et al., 1999, 2002; Ramírez-Amaya and BermúdezRattoni, 1999; Kuo et al., 2001; Chen et al., 2004). Apart from these observations, no systematic approach has been undertaken to elucidate the neural processes underlying conditioning of immune functions. The CTA is a model to study associative learning processes (Berman and Dudai, 2001; Bermúdez-Rattoni, 2004), and it is known to involve a basic neural circuit that includes the nucleus tractus solitari (NTS), parabrachial nucleus (PBN), medial thalamus, amygdala (Am), and insular cortex (IC) (Yamamoto et al., 1994b; Sewards, 2004). In particular, the IC is essential for the acquisition and retention of this associative learning (Bermúdez-Rattoni and McGaugh, 1991; Cubero et al., 


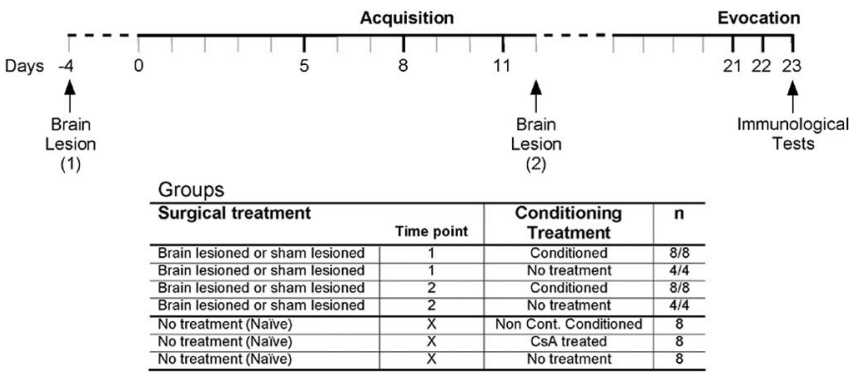

Figure 1. Experimental design. The conditioned groups followed the same fluid restriction protocol consisting of access to fluid consumption in two 15 min sessions (8:00 A.M. and 5:00 P.M.) each day (continuous lines). Water was available ad libitum and is plotted as dashed lines. Three acquisition trials (days 5, 8, and 11) were performed pairing Sac and intraperitoneal CSA injections for the conditioned groups (lesion and sham). The noncontingent conditioned group was exposed to the same stimuli but inversed in order and separated by $9 \mathrm{~h}$. At evocation tests (days 21-23), the conditioned animals were exposed to Sac taste, whereas the noncontingent conditioned animals received water with a subsequent $\mathrm{Sac}$ exposition $9 \mathrm{~h}$ later. The CSA-treated group just received intraperitoneal CsA injections at all acquisition and evocation trials. In contrast, the untreated group followed the fluid restriction plan without further manipulation. The conditioned animals were divided in lesion and sham lesion groups. Brain surgery (IC, Am, or VMH) was performed either before (time point 1, day -4) or after (time point 2, day 12) the acquisition phase.

1999), and it has been postulated that the IC may integrate gustatory and visceral stimuli (Sewards and Sewards, 2001). Moreover, the Am seems to play an important role during formation of CTA (Sewards, 2004), being also relevant for limbic-autonomic interactions (Swanson and Petrovich, 1998). Additionally, the ventromedial nucleus of the hypothalamus (VMH), widely recognized as a satiety center (Vettor et al., 2002), has been proposed to be intimately associated with sympathetic facilitation in peripheral tissues (Saito et al., 1989) including modulation of peripheral immune reactivity (Okamoto et al., 1996).

Thus, given the anatomical and functional relationships among the IC, Am, and VMH, we decided to analyze the role of these brain structures on the learning and memory phases in the Sac-CsA conditioning. Independent experiments were performed for each brain structure, in which excitotoxic lesions were performed before or after the acquisition phase to discriminate between learning and memory processes (see Fig. 1). Surgical and behavioral-appropriate controls were included. At the evocation time, the conditioned response was evaluated at behavioral (CTA) and immunological (splenic T-lymphocyte proliferation and cytokine production) levels.

\section{Materials and Methods}

Animals. Male dark agouti rats (Harlan Laboratories, Borchen, Germany), weighing 220-250 g at the start of the experiments, were housed individually on an inverted $12 \mathrm{~h}$ light/dark schedule (lights on at 7:00 A.M.) with food available ad libitum. Water was available ad libitum, except during the water deprivation regimen. All experimental protocols were performed according to the guidelines of the Institutional Animal Care and the Approval Committees of the Medical Faculty of the University of Duisburg-Essen.

Experimental design. Three independent experiments were performed for each brain structure analyzed: IC, VMH, and Am. Animals were excitotoxically lesioned ( $n=8$, after excluding animals with incorrect placed lesions) or sham lesioned $(n=8)$ in the target brain structure at two different time points: before (time point 1 , day -4 ) or after (time point 2, day 12) the acquisition phase of the conditioning protocol took place (Fig. 1$)$. Three additional control groups $(n=8)$, not receiving any surgical treatment, were included in each experiment: noncontingent conditioned (main conditioning control), CsA treated (pharmacological immunosuppressive control), and untreated (to control handling, stress, and water deprivation regimen effects). Moreover, three additional experiments were performed to control the effects on the basal immune functions of the lesion per se, in which animals $(n=4)$ were surgically manipulated as the groups described above without being conditioned (Fig. 1). These animals were killed 27 (from time point 1) and 11 (from time point 2) days after surgery corresponding to the two lesioning time points in the conditioning experiments.

Conditioning protocol. All animals followed a water deprivation regimen (days $0-12$ and 17-23) consisting of morning (8:00 A.M.) and afternoon (5:00 P.M.) drinking sessions (15 min) each day (Fig. 1). During acquisition (days 5, 8, and 11), the conditioned animals received $0.2 \%$ Sac solution as the CS, paired with intraperitoneal injections of 20 $\mathrm{mg} / \mathrm{kg}$ body weight (b.w.) CsA (LC Laboratories-PKC Pharmaceuticals, Woburn, MA) as the UCS in the morning sessions. In the afternoon sessions, the animals received water paired with an intraperitoneal injection of PBS. The noncontingent conditioned $(n=8)$ and CsA $(n=8)$ groups were exposed to water paired with CsA in the morning and Sac together with PBS injections in the afternoon during acquisition. The evocation phase was performed on days 21-23, when conditioned animals were exposed to Sac during each morning session and were allowed to drink water in the afternoon sessions. The noncontingent conditioned group was exposed to water during each morning session and exposed to Sac during afternoon sessions. The CsA group received water and intraperitoneal CsA injections during the morning sessions and Sac each afternoon session. Untreated animals $(n=8)$ remained unmanipulated just following the same water deprivation regimen. Based on our previous experience with this established model of behavioral conditioned immunosuppression (Exton et al., 1998b, 2000b, 2001), we decided to look for the conditioned immune response after the third evocation trial (day 23); one hour after the animals were deeply anesthetized (isoflurane; CuraMED, Karlsruhe, Germany), the spleens were removed for analysis of lymphocyte proliferation and cytokine production, the brain was removed for histological analysis of the excitotoxic lesions, and blood was withdrawn for differential white blood cell (WBC) counts and flow cytometry analysis.

Brain lesions. Animals were anesthetized by intraperitoneal injection of ketamine ( $0.7 \mathrm{mg} / \mathrm{kg}$ b.w. $)$ and xylazine $(0.3 \mathrm{mg} / \mathrm{kg}$ b.w. $)$. Stereotactic microinjections were performed using Hamilton microsyringes attached to a stereotactic apparatus (David Kopf Instruments, Tujunga, CA) to apply bilateral injections of NMDA solution $(10 \mathrm{mg} / \mathrm{ml}$ dissolved in $0.1 \mathrm{M}$ sterile phosphate buffer, $\mathrm{pH}$ 7.4; Sigma, St. Louis, MO) into the IC [0.8 $\mu \mathrm{l}$; coordinates relative to bregma: anteroposterior (AP), +1.2 ; dorsoventral (DV), -5.5 ; lateral (L), \pm 5.5$]$ and into the Am $(0.6 \mu \mathrm{l}$; coordinates relative to bregma: $\mathrm{AP},-2.5 ; \mathrm{DV},-8.2 ; \mathrm{L}, \pm 5.0$ ). A predominant role of non-NMDA receptors within the VMH in sympathetic facilitation in peripheral tissues has been proved (Narita et al., 1994). Thus, kainic acid (KA; $4.69 \mathrm{mg} / \mathrm{ml}$ dissolved in $0.9 \%$ sterile $\mathrm{NaCl}$ solution; Sigma) was chosen as a neurotoxin to be microinjected into the VMH $(1.0 \mu \mathrm{l}$; coordinates relative to bregma: AP, $-2.8 ; \mathrm{DV},-9.5 ; \mathrm{L}, \pm 0.6)$. Sham-lesioned animals followed the same surgical treatment as the lesioned animals but just received vehicle microinjections (IC and Am, $0.1 \mathrm{M}$ sterile phosphate buffer, $\mathrm{pH}$ 7.4; $\mathrm{VMH}, 0.9 \%$ sterile $\mathrm{NaCl}$ solution). Stereotactic coordinates were obtained from a rat brain stereotactic atlas (Paxinos and Watson, 1998).

Histology. At experimental day 23, all animals were deeply anesthetized (isoflurane), and after the spleen was removed, surgical-treated animals were perfused intracardially with PBS, followed by $4 \%$ paraformaldehyde (Sigma). The brains were then removed and fixed first in paraformaldehyde for $24 \mathrm{~h}$, then in $30 \%$ sucrose until slicing. The brains were cut on a frozen microtome at a thickness of $40 \mu \mathrm{m}$, stained with the standard cresyl violet staining, and analyzed to assess the extent of the lesion using a light microscope (Carl Zeiss, Oberkochen, Germany).

Lymphocyte proliferation assay. Spleen lymphocytes were released from tissue by injecting cell culture medium into the spleen. Cells were washed several times in PBS, pH 7.2, and adjusted to a concentration of $1 \times 10^{6}$ cells $/ \mathrm{ml}$. The cells were then cultured for $72 \mathrm{~h}$ with or without mitogenic stimulation $\left(1.25 \mu \mathrm{g} / \mathrm{ml}\right.$ concanavalin A; Sigma) at $37^{\circ} \mathrm{C}$ in a $5 \% \mathrm{CO}_{2}$ atmosphere. After $48 \mathrm{~h}$ of incubation, the cells were pulsed with $20 \mu \mathrm{l} /$ 
well $\left[{ }^{3} \mathrm{H}\right.$ ] thymidine $(0.5 \mu \mathrm{Ci})$ and harvested $24 \mathrm{~h}$ later. Radioactivity was measured using a beta counter.

Cytokine determination. Spleen lymphocytes were incubated $24 \mathrm{~h}$ with mitogenic stimulation $\left(1.25 \mu \mathrm{g} / \mathrm{ml}\right.$ concanavalin A; Sigma) at $37^{\circ} \mathrm{C}$ in a $5 \% \mathrm{CO}_{2}$ atmosphere. Supernatants from the cell cultures were collected, and IL-2 and IFN- $\gamma$ were assayed using commercial ELISA kits for the detection of rat IL-2 (Biosource, Camarillo, CA) and rat IFN- $\gamma$ (U-CyTech, Utrecht, The Netherlands). Detection limits were $5.0 \mathrm{pg} / \mathrm{ml}$ for IFN- $\gamma$ and $15.6 \mathrm{pg} / \mathrm{ml}$ for IL-2. Samples were analyzed in three different dilutions to ensure linearity of the results.

White blood cell counts. Blood samples were collected in EDTA-coated tubes and analyzed using a differential cell blood counter (Vet abc Animal Blood Counter; Scil, Abx Diagnostics, Montpellier, France)

Flow cytometry. Cell suspensions from the spleen and whole-blood samples were analyzed as described previously (Exton et al., 1998b). Briefly, microtiter plates were pipetted with $1 \times$ $10^{6}$ cells and then incubated with different mouse anti-rat monoclonal antibodies (mAbs) for identification: natural killer (NK) cells (CD161+, TCR-; mAb 3.2.3/mAb R73); T-cytotoxic lymphocytes (CD8+, TCR +; mAb Ox8/mAb R73); T-helper lymphocytes (CD4+, TCR+; mAb W3/25/mAb R73). T-helper lymphocytes were further differentiated into two subsets, naive (CD45+; mAb Ox22) and memory $(\mathrm{CD} 45-)$, as well as B-cells $(\mathrm{CD} 45+, \operatorname{Ig} \kappa$ chain + ; mAb Ox12). A minimum of $1 \times 10^{4}$ events was analyzed on a FACScalibur flow cytometer (Becton Dickinson, Heidelberg, Germany), gating on forward and side-scatter characteristics. All mAbs were purchased from Serotec (Düsseldorf, Germany).

Statistical analyses. An unpaired $t$ test or oneway ANOVA with Fisher's post hoc test was used to examine statistical differences between the groups. Statistical significance was considered when $p$ values were $\leq 0.05$ or $\leq 0.01$.

\section{Results}

\section{Histological analysis}

Histological analysis revealed that bilateral injections of excitotoxin into the IC, Am (NMDA), and VMH (KA) produced a loss of neurons and extensive gliosis in the target tissue (Fig. 2). The IC-induced lesion was located mainly in the medial part of the AP axis of the IC, from the basal part of the parietal cortex to the inferior part of the rhinal sulcus. The NMDA-induced lesions in the IC produced damage to all cortical layers, shrinkage, and holes in the cortical tissue (Fig. 2a). KA-induced lesions in the VMH were located mainly in the dorsomedial part of the VMH (Fig. 2b). Lesions in the Am were located mainly in the basolateral nucleus of the Am (Fig. 2c). Animals with inadequate lesions (i.e., lesions located outside the target area or with no apparent lesions) were discarded from further analyses.
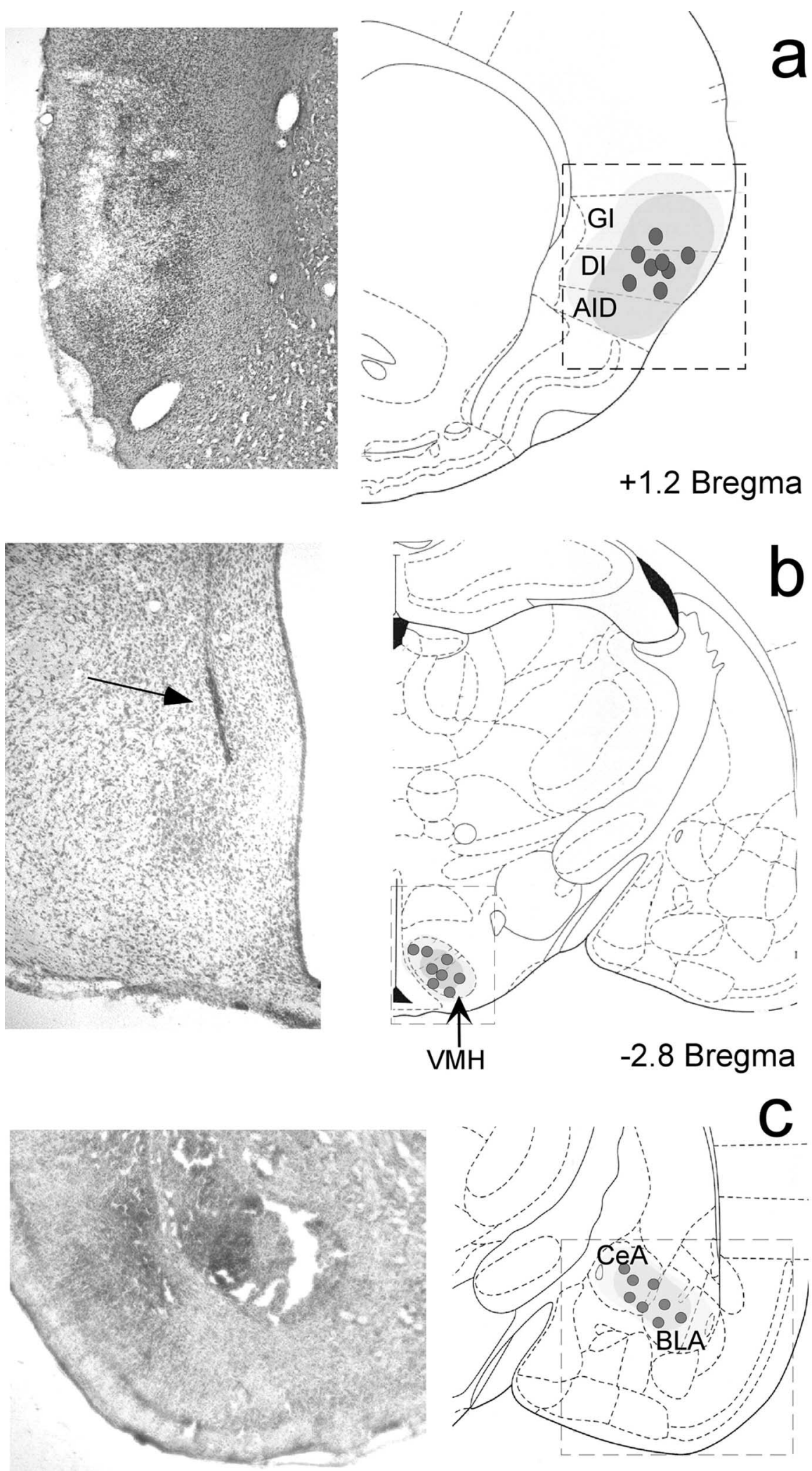

-2.5 Bregma

Figure 2. Excitotoxic lesion sites in coronal sections with cresyl violet stain. The localization of the microsyringe tips (dots) and the approximate extent of the lesion (shadow area) are shown on the right. The insets on the left show photomicrographs $(\boldsymbol{a}, \boldsymbol{c}$, $10 \times ; \boldsymbol{b}, 20 \times)$ of each lesion. $\boldsymbol{a}$, NMDA-induced lesion into the IC. $\boldsymbol{b}$, KA-induced lesion into the VMH. $\boldsymbol{c}$, NMDA-induced lesion into the Am. GI, Granular insular cortex; DI, disgranular insular cortex; AID, agranular insular cortex; CeA, central nucleus of the amygdala; BLA, basolateral nucleus of the amygdala. 
Table 1. Brain lesion effects on cellular immune parameters

\begin{tabular}{|c|c|c|c|c|c|c|c|}
\hline & $\begin{array}{l}\text { Time point } \\
\text { (days after brain lesion) }\end{array}$ & $\begin{array}{l}\text { Surgical } \\
\text { treatment }\end{array}$ & $\begin{array}{l}\text { Proliferation } \\
\text { (percentage relative to naive } \\
\text { control groups) }\end{array}$ & $\begin{array}{l}\text { WBC } \\
\left(10^{3} / \mathrm{mm}^{3}\right)\end{array}$ & $\begin{array}{l}\text { Percentage of blood } \\
\text { lymphocytes }\end{array}$ & $\begin{array}{l}\text { Percentage of blood } \\
\text { monocytes }\end{array}$ & $\begin{array}{l}\text { Percentage of blood } \\
\text { granulocytes }\end{array}$ \\
\hline \multirow[t]{4}{*}{ IC } & \multirow[t]{2}{*}{$1(27)$} & Lesion & $105.5 \pm 2.5$ & $5.6 \pm 1.5$ & $82.9 \pm 2.0$ & $0.5 \pm 0.0$ & $16.6 \pm 2.0$ \\
\hline & & Sham & $100.2 \pm 3.5$ & $5.8 \pm 1.7$ & $80.7 \pm 4.8$ & $0.5 \pm 0.1$ & $18.8 \pm 4.8$ \\
\hline & \multirow[t]{2}{*}{$2(11)$} & Lesion & $111.6 \pm 3.5$ & $14.2 \pm 1.7$ & $58.8 \pm 4.1$ & $0.6 \pm 0.1$ & $40.6 \pm 4.0$ \\
\hline & & Sham & $111.8 \pm 3.2$ & $19.2 \pm 2.2$ & $60.0 \pm 5.9$ & $0.6 \pm 0.1$ & $39.5 \pm 5.8$ \\
\hline \multirow[t]{4}{*}{ VMH } & \multirow[t]{2}{*}{$1(27)$} & Lesion & $125.9 \pm 4.0^{*}$ & $5.1 \pm 0.6$ & $78.8 \pm 2.3$ & $0.5 \pm 0.0$ & $20.7 \pm 2.2$ \\
\hline & & Sham & $102.2 \pm 1.5$ & $5.8 \pm 0.7$ & $83.2 \pm 0.8$ & $0.4 \pm 0.0$ & $16.4 \pm 0.8$ \\
\hline & \multirow[t]{2}{*}{$2(11)$} & Lesion & $98.5 \pm 5.4$ & $19.1 \pm 2.0$ & $54.9 \pm 9.9$ & $0.4 \pm 0.0$ & $44.7 \pm 9.9$ \\
\hline & & Sham & $105.2 \pm 5.5$ & $14.7 \pm 1.1$ & $63.0 \pm 3.9$ & $0.4 \pm 0.0$ & $36.6 \pm 4.0$ \\
\hline \multirow[t]{4}{*}{ Am } & \multirow[t]{2}{*}{$1(27)$} & Lesion & $95.0 \pm 3.5$ & $6.1 \pm 1.2$ & $76.7 \pm 2.5$ & $0.5 \pm 0.1$ & $22.9 \pm 2.5$ \\
\hline & & Sham & $99.8 \pm 1.5$ & $6.1 \pm 0.5$ & $80.2 \pm 1.2$ & $0.5 \pm 0.0$ & $19.4 \pm 1.2$ \\
\hline & \multirow[t]{2}{*}{$2(11)$} & Lesion & $134.6 \pm 4.5^{*}$ & $8.4 \pm 1.0$ & $74.3 \pm 2.1$ & $0.5 \pm 0.0$ & $25.2 \pm 2.1$ \\
\hline & & Sham & $98.5 \pm 3.5$ & $6.5 \pm 1.1$ & $76.8 \pm 3.2$ & $0.4 \pm 0.0$ & $22.9 \pm 3.2$ \\
\hline
\end{tabular}

The WBC profile and the capacity of T-lymphocytes from the spleen to proliferate in response to mitogenic stimulation ( $1.25 \mu \mathrm{g} / \mathrm{ml}$ concanavalin A) were analyzed at two time points after lesioning, corresponding to acquisition ( $27 \mathrm{~d}$ from time point 1 ) and evocation (11 d from time point 2 ) times. Data were analyzed using an unpaired Student's $t$ test. The results are expressed as mean \pm SE. ${ }^{*} p \leq 0.05$ relative to the sham group at each structure and time point ( $n=4$ for all groups).

\section{Brain lesion effects on cellular immune parameters}

To differentiate whether the brain lesions affect the acquisition and/or evocation of the conditioned immunosuppression or whether the lesions per se cause potential effects on the immune response, we evaluated the distribution of the leukocyte subset population and T-lymphocyte proliferation in the spleen 11 and $27 \mathrm{~d}$ after excitotoxic lesions. These time points correspond to the acquisition ( $27 \mathrm{~d}$ from time point 1 ) and evocation ( $11 \mathrm{~d}$ from time point 2) phases on the conditioning protocol (Fig. 1). When the proliferative capacity of splenocytes was tested, a differential effect on the brain areas under investigation was observed (Table 1). The IC lesion did not influence the capacity of splenocytes to proliferate compared with control animals, neither 11 nor $27 \mathrm{~d}$ after lesion.

However, we observed a significant increase $(p \leq 0.05)$ in the proliferation capacity of splenocytes not before $27 \mathrm{~d}$ after lesioning of the VMH (acquisition phase). In contrast, a transient increase in the proliferative capacity of lymphocytes in the spleen was found $11 \mathrm{~d}$ after Am lesion (evocation phase), which returned to control values at $27 \mathrm{~d}$ after lesion (Table 1 ). Total WBC counts performed 11 and $27 \mathrm{~d}$ after lesion revealed no significant changes in the number of WBCs after lesions of the IC, VMH, and Am when compared with sham-treated animals at any time point (Table 1). Flow cytometry analysis showed that none of the brain lesions (IC, VMH, and Am) affect lymphocyte distribution either in the blood or in the spleen at both time points (data not shown).

\section{Brain lesion effects on CTA}

Taste aversion behavior was analyzed in animals with brain lesions performed before conditioning (acquisition), revealing that IC lesions significantly abrogate the CTA $(p \leq 0.05)$. In contrast, animals with lesions of the VMH or the Am did not differ from controls in Sac consumption on the test trial (Fig. 3, left). Analysis of the effects of the brain lesions at the memory stage of CTA (evocation) showed a similar pattern as in the learning stage: animals with IC lesions after acquisition did not develop CTA, whereas animals with lesions of the $\mathrm{VMH}$ and Am displayed a normal CTA like the sham-treated animals (Fig. 3, right). The noncontingent conditioned, CsA-treated, and untreated groups showed a similar water intake at morning $(10.5 \pm 2.5 \mathrm{~g})$ and afternoon (12.5 $\pm 2.5 \mathrm{~g}$ ) drinking sessions (data not shown). A CTA extinction process was observed on the second and third evocation trials (days 22 and 23, respectively) for all conditioned groups. There was no session effect in any group related to the

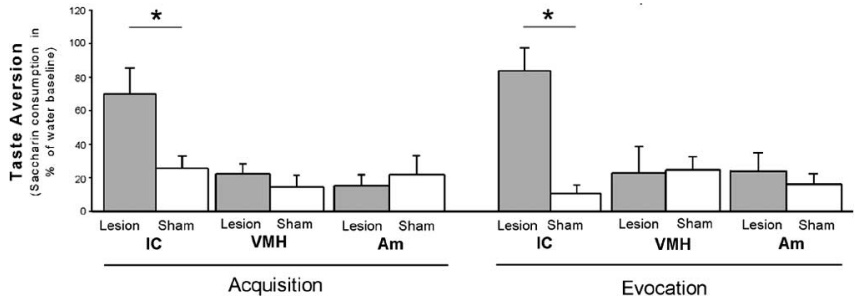

Figure 3. The effects of excitotoxic lesions on CTA, expressed as the percentage of Sac consumption on the first evocation test (day 21) related to the respective fluid baseline consumption (days 18-20). The effects of brain lesions made before (acquisition; left) and after (evocation; right) conditioning took place are shown. Lesion, Excitotoxic lesion; sham, sham lesion. Data were analyzed using a one-way ANOVA, and post hoc analysis was done by Fisher's test. The results are expressed as mean \pm SE. ${ }^{*} p \leq 0.05$ relative to the groups below the line $(n=$ 8 for all groups).

time at which the brain lesions were performed (time point 1, day -4 vs time point 2, day 12 ). On day 23 , both IC-lesioned groups drank the Sac solution almost at $100 \%$ of water baseline (IC lesion time point 1, $98.8 \pm 7.5 \%$; IC lesion time point 2, $102.8 \pm$ $12.5 \%)$. The Sac consumption on day 23 for the other conditioned groups was between 59 and $75 \%$ of water baseline consumption (data not shown).

\section{Brain lesion effects on behavioral conditioned suppression of} cellular immune function

In vitro proliferative capacity of spleen lymphocytes and cytokine production (IL-2 and IFN- $\gamma$ ) was analyzed in animals with brain lesions performed before and after acquisition. Lesions of the IC as well as the Am before acquisition completely abrogated the conditioned suppressive response measured as lymphocyte proliferation (Fig. 4a, left) and production of IL-2 (Fig. 4b, left) and IFN- $\gamma$ (Fig. 4c left). In contrast, animals with lesions on the VMH did not differ from animals with sham lesions in their behaviorally conditioned immune response (Fig. $4 a-c$, left). A different pattern was observed with brain lesions generated at the evocation stage of conditioned immunosuppression. IC as well as $\mathrm{VMH}$ lesions completely abrogated the behavioral conditioned suppression of spleen lymphocytes to proliferate (Fig. $4 a$, right) as well as the conditioned effects on IL-2 (Fig. $4 b$, right) and IFN- $\gamma$ (Fig. $4 c$, right) secretion. In contrast, lesions performed in the Am did not affect the recall of the conditioned immunosuppression (Fig. $4 a-c$, right). The untreated control groups from all 

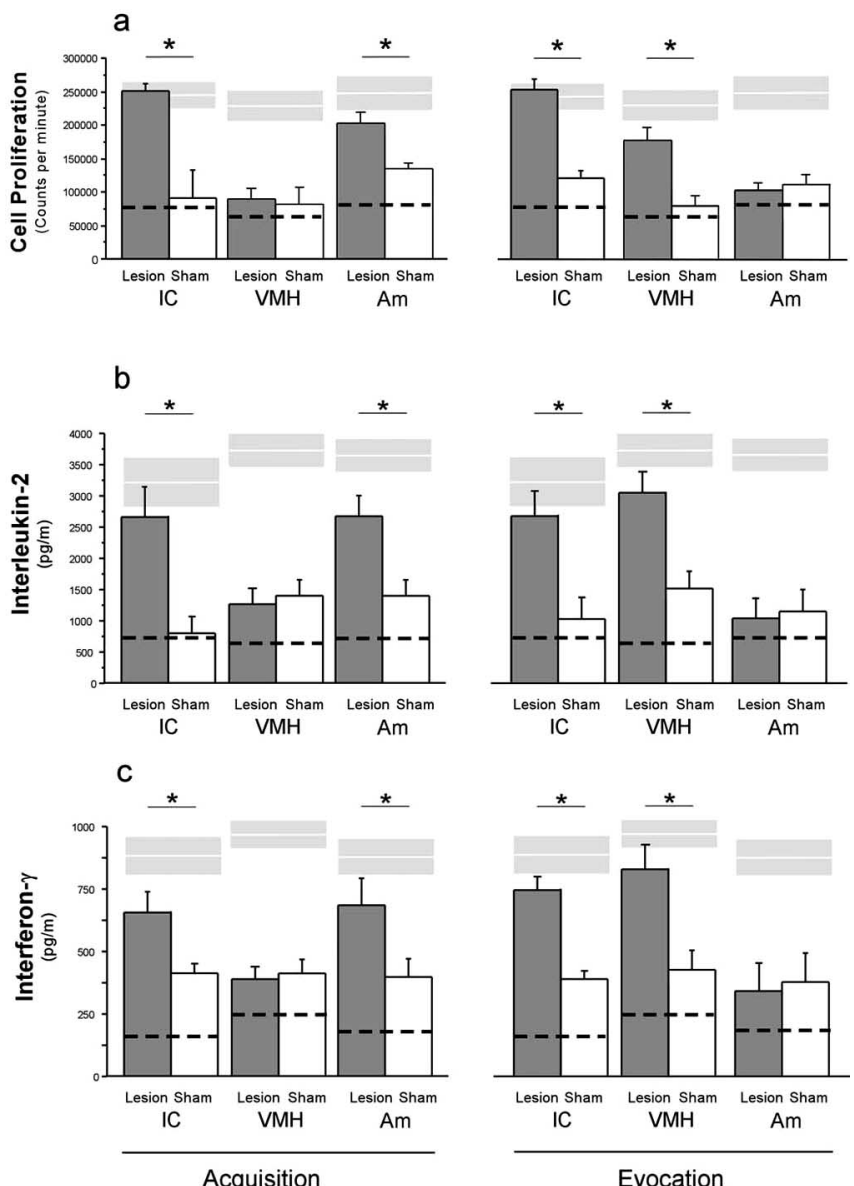

Figure 4. The effects of excitotoxic lesions on conditioned immunosuppresion were analyzed after the last evocation test (day 23) of three independent experiments, each one targeting one brain structure (IC, Am, or VMH). On the left is shown the effects of brain lesions performed before conditioning took place (Acquisition) and after on the right side (Evocation). $\boldsymbol{a}$ shows the capacity of T-lymphocytes from the spleen to proliferate in response to mitogenic stimulation (1.25 $\mu \mathrm{g} / \mathrm{ml}$ concanavalin A). $\boldsymbol{b}$ and $\boldsymbol{c}$ show the interleukin-2 and interferon- $\gamma$ levels from supernatant samples of the same cell cultures used for $\boldsymbol{a}$. Dashed lines depict the mean value for CsA-treated groups, and white lines within gray boxes depict mean \pm SE of the noncontingent conditioned groups. Lesion, Excitotoxic lesion; sham, sham lesion. Data were analyzed using a one-way ANOVA, and post hoc analysis was done by Fisher's test. The results in bars are expressed as the mean $\pm S E .{ }^{*} p \leq 0.05$ relative to the groups below the line ( $n=8$ for all groups).

experiments showed similar proliferative capacity (IC lesion, $225.2 \pm 25.3$; Am lesion, $215.6 \pm 18.9$; VMH lesion, $239.1 \pm 38.3$; $\left.\times 10^{3} \mathrm{cpm}\right)$ as the noncontingent conditioned animals. Flow cytometry analysis showed that the brain lesions (IC, VMH, and Am) did not affect lymphocyte distribution in the blood nor in the spleen at both time points (data not shown).

\section{Discussion}

The ability of the CNS to modulate peripheral immune functions has been clearly demonstrated through classical conditioning paradigms (Ader and Cohen, 2000; Exton et al., 2001). However, not much is known about the central processes underlying behavioral conditioned changes in immune function. Thus, we decided to approach this issue by analyzing learning and memory processes of behavioral conditioned immunosuppression by using excitotoxic brain lesions before or after the acquisition phase. The current results reveal that different neural circuits process the behavioral and immunological components of the condi- tioned response. CTA was disrupted by IC lesions performed before and after acquisition, whereas Am and VMH lesions did not affect CTA. A different pattern was observed in the behavioral conditioned immune response, measured as the capacity of spleen T-lymphocytes to proliferate and to produce cytokines (IL-2 and IFN- $\gamma$ ). The IC was found to be essential to acquire and evoke the conditioned immune suppression, whereas the Am appeared necessary only for the acquisition of this associative learning. In contrast, the VMH lesion disrupted the conditioned immune response.

The present results confirm the essential role of the IC acquiring and retrieving CTA (Bermúdez-Rattoni and McGaugh, 1991; Cubero et al., 1999). It has been postulated that the role of the IC within CTA should relate to the associative process rather than to just a sensing activity, because the integrity of the IC is not necessary for taste responsiveness or discrimination (Kiefer and Braun, 1977; Grill and Norgren, 1978).

Although the Am receives several projections from gustatory and visceral brainstem structures (Ricardo and Koh, 1978; Wallace et al., 1989), and a strong amgydalo-insula-cortical interaction has been demonstrated (Shi and Cassell, 1998), the present data support early observations that lesions of the Am have essentially no disruptive effects on acquisition and evocation of CTA (Bermúdez-Rattoni and McGaugh, 1991; Galaverna et al., 1993; Schafe et al., 1998). Finally, our results show that lesions of the VMH had essentially no effects on the acquisition and evocation of CTA, although this hypothalamic nucleus regulates feeding behavior; this is consistent with previous reports (Kramer et al., 1983; Yamamoto et al., 1995). Additionally, with the exception of the IC-lesioned groups, all other conditioned groups displayed similar CTA extinction processes, indicating that the CTA strength was not affected by these brain lesions (Mikulka and Klein, 1980; Taylor and Boakes, 2002).

Our well established model of behavioral conditioned immunosuppression (Exton et al., 2000b, 2001) enabled us to analyze the role of the IC, Am, and VMH in the conditioned behavioral immune response. Excitotoxic brain lesions differentially affected the behavioral conditioned immunosuppressive response, measured as spleen lymphocyte proliferative capacity and cytokine (IL-2 and IFN- $\gamma$ ) production. The results demonstrate that the IC is necessary for the CS-UCS association and evocation (possibly as a storage site) of the conditioned immunosuppression. This is in agreement with previous observations showing that the IC is involved at the acquisition and at evocation times of the conditioned changes of antibody production (RamírezAmaya et al., 1996, 1998; Ramírez-Amaya and BermúdezRattoni, 1999; Chen et al., 2004).

Am lesions abrogate the conditioned immunosuppression when lesions were performed before CS-UCS pairing. This effect cannot be explained by the role of the Am in the gustatory input, because lesions of this structure did not affect CTA. Moreover, lesions performed after conditioning did not affect the conditioned immunosuppressive effects, excluding the possibility that Am lesions affect taste perception. The present results, together with the fact that the Am has several visceral afferent projections (Ricardo and Koh, 1978) that can be activated by different peripheral immune stimuli (Elmquist et al., 1993; Kusnecov et al., 1999; Pacheco-López et al., 2002), indicate that the Am could mediate the immune-visceral input induced by the intraperitoneal CsA injection.

In contrast to the Am, VMH lesions affected the conditioned immune response only when it was performed after the acquisition time. It has been demonstrated that VMH lesions decrease 
NK cell activity in the spleen (Forni et al., 1983; Katafuchi et al., 1994), and electrical stimulation of this hypothalamic nucleus reduces the capacity of lymphocytes in the spleen to proliferate (Okamoto et al., 1996). We have previously demonstrated that behaviorally conditioned immunosuppression is merely mediated via neural innervation of the spleen, implicating an indispensable role of the sympathetic outflow at the evocation time (Exton et al., 1999, 2002). Importantly, neuroanatomical and electrophysiological data indicate that the IC modulates sympathetic nervous activity (Oppenheimer et al., 1992) via obligatory synapses in the lateral hypothalamic area (LHA) (Cechetto and Chen, 1990, 1992; Butcher and Cechetto, 1998). Additionally, the fact that no direct projections have been reported from the IC to the VMH, together with the intrahypothalamic LHA-VMH reciprocal interactions reported (Luiten and Room, 1980; Ter Horst and Luiten, 1987), indicate that at least one additional brain structure, potentially the LHA, could be involved in the efferent pathway of behavioral conditioned immunosuppression. It is important to mention that the histological analysis did not show the VMH lesions to affect surrounding hypothalamic nuclei (e.g., paraventricular hypothalamic nuclei, LHA). However, we cannot discard that lesioning the VMH could induce intrahypothalamic disruption, resulting in a neuroendocrine deregulation with its concomitant peripheral immune system effects (Webster et al., 2002) and thus partially affecting the present results.

Recently, a neocortical-immune axis as a neural efferent pathway has been proposed, giving additional support to the brain abilities to modulate peripheral immune functions (Moshel et al., 2005; Tuohy, 2005). In this regard, we propose that the IC is one of the main neocortices with immunomodulatory capacities, with hypothalamic relays (VMH/LHA) and sympathetic peripheral mechanisms.

Neural pathways related to learning-memory formation are considered as plastic and dynamic networks and not as static wiring models, because feedback and feedforward interactions are known to exist in learning and specifically within the CTA circuitry (Katz et al., 2002; Lundy and Norgren, 2004). Furthermore, a hedonic neural circuit independent from sensory processes seems to be modified during CTA formation (Yamamoto et al., 1994a; Grancha et al., 2002). We observed that the brain structures studied are differentially involved in eliciting the behavioral and the immunological component of the conditioned response. Thus, we propose the existence of different neural circuits processing each modality of the conditioned response (behavioral vs immune). However, to unravel the complete neural circuitry in the context of behavioral conditioned immune changes, additional experiments are needed that target brain stem nuclei such as the NTS and the PBN, as well as the LHA.

To differentiate the effects of different lesions related to behavioral immune conditioning from potential effects on the immune response induced by the lesions per se, cellular immune parameters were assessed at two time points after lesioning, corresponding to acquisition ( $27 \mathrm{~d}$ from time point 1 ) and evocation (11 d from time point 2). These data demonstrate that IC lesions affect neither cellular immune responses nor the WBC profile. In contrast, a minimal but significant enhanced capacity of spleen lymphocytes to proliferate was observed $11 \mathrm{~d}$ after Am lesion and $27 \mathrm{~d}$ after $\mathrm{VMH}$ lesion. However, these time points do not correspond with the time points at which conditioned immunosuppression was abrogated (Am, $27 \mathrm{~d}$; VMH, $11 \mathrm{~d}$ ), demonstrating that the outcome of brain lesions observed in the conditioned animals represents specific conditioning effects. Furthermore,

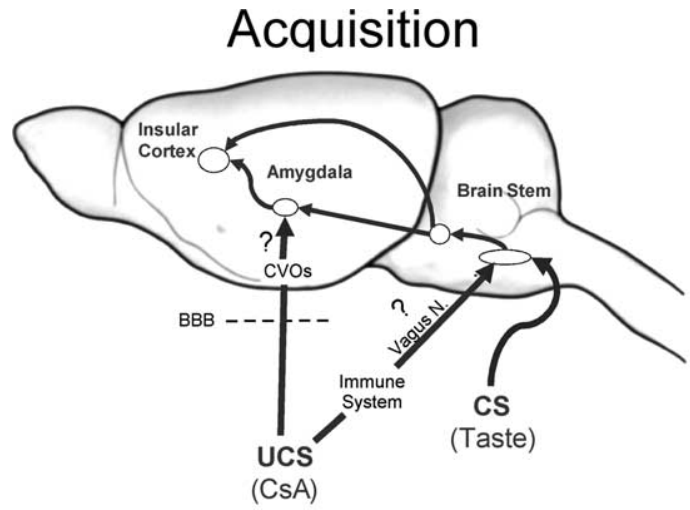

Evocation

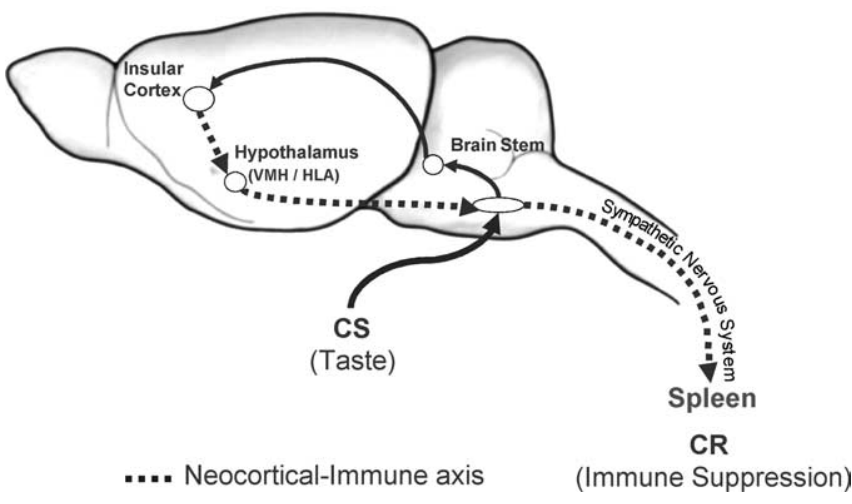

Figure 5. Conditioned immunosuppression model. The present results indicate that the IC may play an associative role being necessary to acquire and evoke the behavioral conditioned immune response. The Am seems to mediate the input of visceral information that is required at the acquisition time, whereas VMH lesions disrupt the brain to immune communication necessary to evoke the immunological conditioned response. At the acquisition time, there are two main possible afferent routes for the UCS (intraperitoneal CSA): indirect afferent route, the CSA may disrupt the peripheral cytokine homeostasis, which could be detected by the CNS immunesensing capacities (e.g., vagus nerve); direct afferent route, the CsA might reach the CNS through those brain areas with a weak $B B B$, like circumventricular organs, subsequently signaling to forebrain structures. At the evocation time, the neocortical-immune axis, with hypothalamic relays (VMH/LHA) and sympathetic peripheral mechanism, seems to be the efferent pathway through which the brain is able to modify peripheral immune functions. CR, Conditioned response; CVOs, circumventricular organs.

the effects of brain lesions on the conditioned inhibition of T-cell proliferation and cytokine production are not related to changes in the circulation of lymphocyte subsets. In addition, we have previously demonstrated that the behavioral conditioned immune response in the spleen is not dependent on changes in lymphocyte subset distribution (Exton et al., 2001).

How, when, and where CsA, used in this model as an UCS, is detected by the CNS is still unknown. The main immunological effect of CsA is to inhibit the production of cytokines such as IL-2, $\gamma$-IFN, IL-4, granulocyte-macrophage colony-stimulating factor, and tumor necrosis factor- $\alpha$ (Schumacher and Nordheim, 1992), with its concomitant suppression of the T-lymphocyte capacity to proliferate. Therefore, it might be possible that CsA disrupts the peripheral cytokine homeostasis, which could be detected by the CNS immune-sensing capacities such as the vagus nerve, the NTS, or forebrain structures (Watkins et al., 1995; Goehler et al., 2000). In addition, although under normal conditions CsA has not been reported to cross the blood-brain barrier 
(BBB) during acute administration (Fahr, 1993), direct effects on the hypothalamus and the pituitary have been reported (Stephanou et al., 1992; Esquifino et al., 1995; Wera et al., 1995; Chou and Fong, 1999; López-Calderón et al., 1999). Thus, CsA might reach the CNS through those brain areas with a weak BBB, like circumventricular organs, subsequently signaling to forebrain structures (Fig. 5). An additional systematic analysis will be necessary to elucidate the afferent pathway(s) and messenger(s) used by CsA to reach the brain.

In summary, our data reveal relevant neural mechanisms underlying the learning and memory processes of behavioral conditioned immunosuppression (Fig. 5). The results demonstrate that IC lesions performed before and after acquisition disrupt the behavioral component of the conditioned response. In contrast, Am and VMH lesions do not affect CTA behavior. The behavioral conditioned immunosuppression was differentially affected by the excitotoxic lesions. Here, the IC may play an associative role being necessary to acquire and evoke the behavioral conditioned immune response. The Am seems to mediate the input of visceral information that is required at the acquisition time, whereas the $\mathrm{VMH}$ lesion seems to disrupt the brain to immune communication necessary to evoke the immunological conditioned response. The neocortical-immune axis, with hypothalamic relays (VMH/ LHA) and sympathetic peripheral mechanisms, seems to be the efferent pathway through which the brain is able to modify peripheral immune functions at the evocation time during behavioral immunoconditioning.

\section{References}

Ader R, Cohen N (2000) Conditioning and immunity. In: Psychoneuroimmunology (Ader R, Felten DL, Cohen N, eds), pp 3-34. San Diego: Academic.

Berman DE, Dudai Y (2001) Memory extinction, learning anew, and learning the new: dissociations in the molecular machinery of learning in cortex. Science 291:2417-2419.

Bermúdez-Rattoni F (2004) Molecular mechanisms of taste-recognition memory. Nat Rev Neurosci 5:209-217.

Bermúdez-Rattoni F, McGaugh JL (1991) Insular cortex and amygdala lesions differentially affect acquisition on inhibitory avoidance and conditioned taste aversion. Brain Res 549:165-170.

Butcher KS, Cechetto DF (1998) Receptors in lateral hypothalamic area involved in insular cortex sympathetic responses. Am J Physiol 275:H689-H696.

Cechetto DF, Chen SJ (1990) Subcortical sites mediating sympathetic responses from insular cortex in rats. Am J Physiol 258:R245-R255.

Cechetto DF, Chen SJ (1992) Hypothalamic and cortical sympathetic responses relay in the medulla of the rat. Am J Physiol 263:R544-R552.

Chen J, Lin W, Wang W, Shao F, Yang J, Wang B, Kuang F, Duan X, Ju G (2004) Enhancement of antibody production and expression of c-Fos in the insular cortex in response to a conditioned stimulus after a single-trial learning paradigm. Behav Brain Res 154:557-565.

Chou YC, Fong JC (1999) Cyclosporin A induces a biphasic increase in $\mathrm{KCl}$-induced calcium influx in $\mathrm{GH} 3$ pituitary cells. Biochem Biophys Res Commun 254:169-173.

Cubero I, Thiele TE, Bernstein IL (1999) Insular cortex lesions and taste aversion learning: effects of conditioning method and timing of lesion. Brain Res 839:323-330.

Dantzer R, Konsman JP, Bluthe RM, Kelley KW (2000) Neural and humoral pathways of communication from the immune system to the brain: parallel or convergent? Auton Neurosci 85:60-65.

Elmquist JK, Ackermann MR, Register KB, Rimler RB, Ross LR, Jacobson CD (1993) Induction of Fos-like immunoreactivity in the rat brain following Pasteurella multocida endotoxin administration. Endocrinology 133:3054-3057.

Elmquist JK, Scammell TE, Saper CB (1997) Mechanisms of CNS response to systemic immune challenge: the febrile response. Trends Neurosci 20:565-570

Esquifino AI, Moreno ML, Arce A, Agrasal C, Pérez-Díaz J, Villanua MA
(1995) Effects of cyclosporine at the hypothalamic-pituitary axis in pituitary-grafted young female rats. J Endocrinol 144:159-164.

Exton MS, Schult M, Donath S, Strubel T, Nagel E, Westermann J, Schedlowski M (1998a) Behavioral conditioning prolongs heart allograft survival in rats. Transplant Proc 30:2033.

Exton MS, von Horsten S, Schult M, Voge J, Strubel T, Donath S, Steinmuller C, Seeliger H, Nagel E, Westermann J, Schedlowski M (1998b) Behaviorally conditioned immunosuppression using cyclosporine A: central nervous system reduces IL-2 production via splenic innervation. J Neuroimmunol 88:182-191.

Exton MS, von Horsten S, Voge J, Westermann J, Schult M, Nagel E, Schedlowski M (1998c) Conditioned taste aversion produced by cyclosporine A: concomitant reduction in lymphoid organ weight and splenocyte proliferation. Physiol Behav 63:241-247.

Exton MS, Schult M, Donath S, Strubel T, Bode U, del Rey A, Westermann J, Schedlowski M (1999) Conditioned immunosuppression makes subtherapeutic cyclosporin effective via splenic innervation. Am J Physiol 276:R1710-R1717.

Exton MS, Elfers A, Jeong WY, Bull DF, Westermann J, Schedlowski M (2000a) Conditioned suppression of contact sensitivity is independent of sympathetic splenic innervation. Am J Physiol Regul Integr Comp Physiol 279:R1310-R1315.

Exton MS, von A, Buske K, Stockhorst U, Gobel U, Schedlowski M (2000b) Pavlovian conditioning of immune function: animal investigation and the challenge of human application. Behav Brain Res 110:129-141.

Exton MS, Herklotz J, Westermann J, Schedlowski M (2001) Conditioning in the rat: an in vivo model to investigate the molecular mechanisms and clinical implications of brain-immune communication. Immunol Rev 184:226-235.

Exton MS, Gierse C, Meier B, Mosen M, Xie Y, Frede S, Goebel MU, Limmroth V, Schedlowski M (2002) Behaviorally conditioned immunosuppression in the rat is regulated via noradrenaline and beta-adrenoceptors. J Neuroimmunol 131:21-30.

Fahr A (1993) Cyclosporin clinical pharmacokinetics. Clin Pharmacokinet 24:472-495.

Forni G, Bindoni M, Santoni A, Belluardo N, Marchese AE, Giovarelli M (1983) Radiofrequency destruction of the tuberoinfundibular region of hypothalamus permanently abrogates NK cell activity in mice. Nature 306:181-184.

Galaverna OG, Seeley RJ, Berridge KC, Grill HJ, Epstein AN, Schulkin J (1993) Lesions of the central nucleus of the amygdala. I: Effects on taste reactivity, taste aversion learning and sodium appetite. Behav Brain Res 59:11-17.

Garcia J, Lasiter PS, Bermúdez-Rattoni F, Deems DA (1985) A general theory of aversion learning. Ann NY Acad Sci 443:8-21.

Goehler LE, Gaykema RP, Hansen MK, Anderson K, Maier SF, Watkins LR (2000) Vagal immune-to-brain communication: a visceral chemosensory pathway. Auton Neurosci 85:49-59.

Grancha ML, Navarro M, Cubero I, Thiele TE, Bernstein IL (2002) Induction of a brainstem correlate of conditioned taste aversion expression: role of the pontine parabrachial nucleus. Behav Brain Res 131:205-209.

Grill HJ, Norgren R (1978) Chronically decerebrate rats demonstrate satiation but not bait shyness. Science 201:267-269.

Hsueh CM, Kuo JS, Chen SF, Huang HJ, Cheng FC, Chung LJ, Lin RJ (1999) Involvement of catecholamines in recall of the conditioned NK cell response. J Neuroimmunol 94:172-181.

Hsueh CM, Chen SF, Lin RJ, Chao HJ (2002) Cholinergic and serotonergic activities are required in triggering conditioned NK cell response. J Neuroimmunol 123:102-111.

Katafuchi T, Okada E, Take S, Hori T (1994) The biphasic changes in splenic natural killer cell activity following ventromedial hypothalamic lesions in rats. Brain Res 652:164-168.

Katz DB, Nicolelis MA, Simon SA (2002) Gustatory processing is dynamic and distributed. Curr Opin Neurobiol 12:448-454.

Kiefer SW, Braun JJ (1977) Absence of differential associative responses to novel and familiar taste stimuli in rats lacking gustatory neocortex. J Comp Physiol Psychol 91:498-507.

Kramer TH, Sclafani A, Kindya K, Pezner M (1983) Conditioned taste aversion in lean and obese rats with ventromedial hypothalamic knife cuts. Behav Neurosci 97:110-119.

Kuo JS, Chen SF, Huang HJ, Yang CS, Tsai PJ, Hsueh CM (2001) The in- 
volvement of glutamate in recall of the conditioned NK cell response. J Neuroimmunol 118:245-255.

Kusnecov AW, Liang R, Shurin G (1999) T-lymphocyte activation increases hypothalamic and amygdaloid expression of CRH mRNA and emotional reactivity to novelty. J Neurosci 19:4533-4543.

López-Calderón A, Soto L, Villanua MA, Vidarte L, Martín AI (1999) The effect of cyclosporine administration on growth hormone release and serum concentrations of insulin-like growth factor-I in male rats. Life Sci 64:1473-1483.

Luiten PG, Room P (1980) Interrelations between lateral, dorsomedial and ventromedial hypothalamic nuclei in the rat. An HRP study. Brain Res 190:321-332.

Lundy RF, Norgren R (2004) Activity in the hypothalamus, amygdala, and cortex generates bilateral and convergent modulation of pontine gustatory neurons. J Neurophysiol 91:1143-1157.

Mikulka P, Klein S (1980) Resistance to extinction of a taste aversion: effects of level of training and procedures used in acquisition and extinction. Am J Psychol 93:631-641.

Moshel YA, Durkin HG, Amassian VE (2005) Lateralized neocortical control of T lymphocyte export from the thymus I. Increased export after left cortical stimulation in behaviorally active rats, mediated by sympathetic pathways in the upper spinal cord. J Neuroimmunol 158:3-13.

Narita K, Nishihara M, Takahashi M (1994) Concomitant regulation of running activity and metabolic change by the ventromedial nucleus of the hypothalamus. Brain Res 642:290-296.

Okamoto S, Ibaraki K, Hayashi S, Saito M (1996) Ventromedial hypothalamus suppresses splenic lymphocyte activity through sympathetic innervation. Brain Res 739:308-313.

Oppenheimer SM, Saleh TM, Wilson JX, Cechetto DF (1992) Plasma and organ catecholamine levels following stimulation of the rat insular cortex. Brain Res 569:221-228.

Pacheco-López G, Espinosa E, Zamorano R, Ramírez-Amaya V, BermúdezRattoni F (2002) Peripheral protein immunization induces rapid activation of the CNS, as measured by c-Fos expression. J Neuroimmunol 131:50-59.

Paxinos G, Watson S (1998) The rat brain in stereotaxic coordinates. San Diego: Academic.

Ramírez-Amaya V, Bermúdez-Rattoni F (1999) Conditioned enhancement of antibody production is disrupted by insular cortex and amygdala but not hippocampal lesions. Brain Behav Immun 13:46-60.

Ramírez-Amaya V, Alvarez-Borda B, Ormsby CE, Martinez RD, Perez M, Bermúdez-Rattoni F (1996) Insular cortex lesions impair the acquisition of conditioned immunosuppression. Brain Behav Immun 10:103-114

Ramírez-Amaya V, Alvarez-Borda B, Bermudez-Rattoni F (1998) Differential effects of NMDA-induced lesions into the insular cortex and amygdala on the acquisition and evocation of conditioned immunosuppression. Brain Behav Immun 12:149-160.

Ricardo JA, Koh ET (1978) Anatomical evidence of direct projections from the nucleus of the solitary tract to the hypothalamus, amygdala, and other forebrain structures in the rat. Brain Res 153:1-26.

Saito M, Minokoshi Y, Shimazu T (1989) Accelerated norepinephrine turn- over in peripheral tissues after ventromedial hypothalamic stimulation in rats. Brain Res 481:298-303.

Schafe GE, Thiele TE, Bernstein IL (1998) Conditioning method dramatically alters the role of amygdala in taste aversion learning. Learn Mem 5:481-492.

Schumacher A, Nordheim A (1992) Progress towards a molecular understanding of cyclosporin A-mediated immunosuppression. Clin Investig 70:773-779.

Sewards TV (2004) Dual separate pathways for sensory and hedonic aspects of taste. Brain Res Bull 62:271-283.

Sewards TV, Sewards MA (2001) Cortical association areas in the gustatory system. Neurosci Biobehav Rev 25:395-407.

Shi CJ, Cassell MD (1998) Cortical, thalamic, and amygdaloid connections of the anterior and posterior insular cortices. J Comp Neurol 399:440-468.

Stephanou A, Sarlis NJ, Knight RA, Lightman SL, Chowdrey HS (1992) Effects of cyclosporine A on the hypothalamic-pituitary-adrenal axis and anterior pituitary interleukin-6 mRNA expression during chronic inflammatory stress in the rat. J Neuroimmunol 41:215-222.

Swanson LW, Petrovich GD (1998) What is the amygdala? Trends Neurosci 21:323-331.

Taylor KM, Boakes RA (2002) Extinction of conditioned taste aversions: effects of concentration and overshadowing. Q J Exp Psychol B 55:213-239.

Ter Horst GJ, Luiten PG (1987) Phaseolus vulgaris leuco-agglutinin tracing of intrahypothalamic connections of the lateral, ventromedial, dorsomedial and paraventricular hypothalamic nuclei in the rat. Brain Res Bull 18:191-203.

Tracey K (2002) The inflammatory reflex. Nature 420:853-859.

Tuohy VK (2005) The neocortical-immune axis. J Neuroimmunol 158:1-2.

Vettor R, Fabris R, Pagano C, Federspil G (2002) Neuroendocrine regulation of eating behavior. J Endocrinol Invest 25:836-854.

Wallace DM, Magnuson DJ, Gray TS (1989) The amygdalo-brainstem pathway: selective innervation of dopaminergic, noradrenergic and adrenergic cells in the rat. Neurosci Lett 97:252-258.

Watkins LR, Maier SF, Goehler LE (1995) Cytokine-to-brain communication: a review and analysis of alternative mechanisms. Life Sci 57:1011-1026.

Webster JI, Tonelli L, Sternberg EM (2002) Neuroendocrine regulation of immunity. Annu Rev Immunol 20:125-163.

Wera S, Zheng L, Hooghe-Peters EL, Belayew A, Martial JA, Velkeniers B (1995) Cyclosporin A, rapamycin and FK506 decrease prolactin release from rat pituitary cells in primary culture. Endocr Res 21:623-633.

Yamamoto T, Shimura T, Sakai N, Ozaki N (1994a) Representation of hedonics and quality of taste stimuli in the parabrachial nucleus of the rat. Physiol Behav 56:1197-1202.

Yamamoto T, Shimura T, Sako N, Yasoshima Y, Sakai N (1994b) Neural substrates for conditioned taste aversion in the rat. Behav Brain Res 65:123-137.

Yamamoto T, Fujimoto Y, Shimura T, Sakai N (1995) Conditioned taste aversion in rats with excitotoxic brain lesions. Neurosci Res 22:31-49. 